\title{
Aberrant expression and hormonal regulation of Galectin-3 in endometriosis women with infertility
}

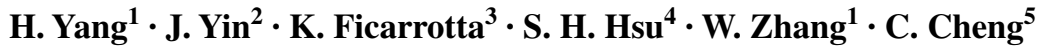

Received: 2 September 2015 / Accepted: 21 January 2016 / Published online: 17 February 2016

(C) The Author(s) 2016. This article is published with open access at Springerlink.com

\begin{abstract}
Objective To investigate the role and potential molecular mechanism of Galectin-3 (Gal-3) in the etiology of endometriosis-associated infertility.

Methods We detected Gal-3 expression in eutopic endometrium from women with endometriosis-associated infertility and healthy women without endometriosis or infertility. We then evaluated Gal-3 expression in endometrial glandular epithelial cells (EECs) and endometrial stromal cells (ESCs) and investigated its response to hormone stimulation in EECs and ESCs from both groups of women.

Results Results of real-time PCR and western blot analysis showed Gal-3 expression in both proliferative and secretory stages of the menstrual cycle decreased significantly in women with endometriosis-associated infertility compared to healthy
\end{abstract}

H. Yang and J. Yin contributed equally to this work.

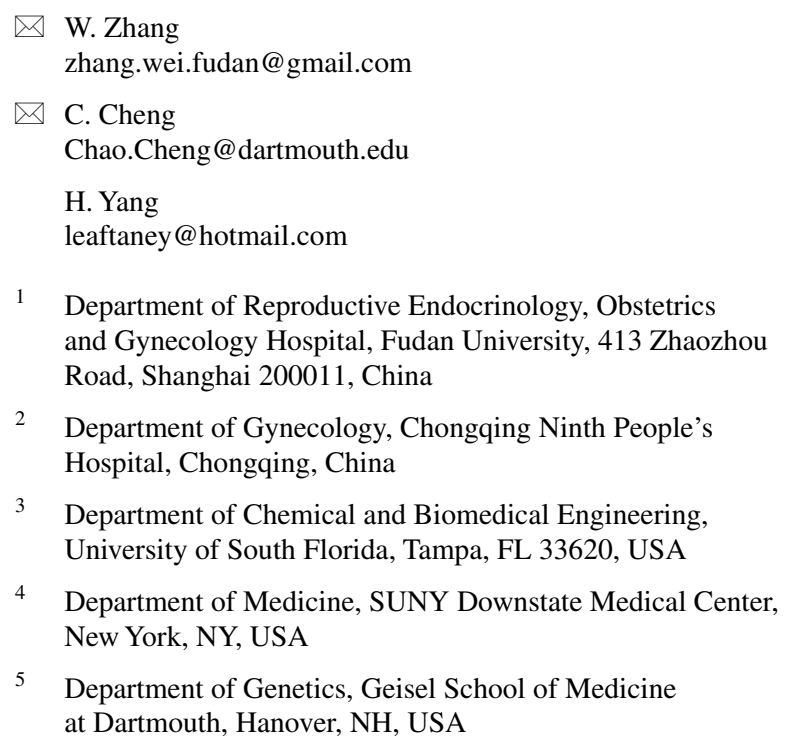

1 Department of Reproductive Endocrinology, Obstetrics and Gynecology Hospital, Fudan University, 413 Zhaozhou Road, Shanghai 200011, China

2 Department of Gynecology, Chongqing Ninth People's Hospital, Chongqing, China

3 Department of Chemical and Biomedical Engineering, University of South Florida, Tampa, FL 33620, USA

4 Department of Medicine, SUNY Downstate Medical Center, New York, NY, USA

5 Department of Genetics, Geisel School of Medicine at Dartmouth, Hanover, NH, USA

women. The changes in expression of Gal-3 were more dramatic in EECs than ESCs. Moreover, estrogen (E2) and progesterone (P4) induced Gal-3 expression in EECs of healthy groups, and $\mathrm{P} 4$ was more significant than $\mathrm{E} 2$ and combined $\mathrm{E} 2$ and $\mathrm{P} 4$ (E2P4). However, in the endometriosis group, $\mathrm{P} 4$ failed to induce a similar increase in Gal-3 expression.

Conclusions Our results suggest that aberrant expression of Gal-3 might contribute to infertility in patients with endometriosis due to progesterone resistance.

Keywords Endometriosis · Galectin-3 (Gal-3) - Uterine receptivity $\cdot$ Sex hormone

\section{Introduction}

Endometriosis is a benign gynecological disorder with malignant biological characteristics and is defined by the presence of endometrial glands and stroma outside the uterine cavity. Ectopic endometrial cells can spread to pelvic organs such as rectum, bladder, and ovaries. This spreading contributes to various clinical manifestations including irregular uterine bleeding, dyspareunia, chronic pelvic pain, and infertility: a condition affecting $10 \%$ of women of reproductive age [1-3]. Statistically, 30-50\% of females with endometriosis are infertile and $25-50 \%$ of females with infertility have endometriosis [4]. Although the association between endometriosis and infertility has been well established, the underlying mechanisms remain unknown.

It has been proposed that abnormal endometrial development in patients with endometriosis contributes to endometriosis-related infertility [5]. It is well known that endometrial receptivity is vital in women's fertility. Human tissue and mouse model studies have demonstrated that the molecular markers of endometrial receptivity are altered 
in both humans and mice with endometriosis. Integrins, matrix metalloproteinases (MMPs), and homeobox genes (HoxA10) display aberrant expression patterns in the eutopic endometrium of women with endometriosis [6, 7]. Other alterations of biochemical or molecular markers have also been reported, including changes in the levels of vascular endothelial growth factor (VEGF) [8] and interleukin 6 (IL-6) [9]. Galectin-3 (Gal-3), a $\beta$-galactosidebinding protein, has been related to endometrial receptivity during embryonic implantation recently $[10,11]$. Gal-3 with a $\sim 31 \mathrm{kDa}$ lectin contains a collagen- $\alpha$-like domain, an N-terminal domain, and the carbohydrate recognition domain (CRD) [12]. These three structures allow Gal-3 to possess specific biological functions, including cell adhesion, migration, cell-extracellular matrix interactions [13], immune response, [14] and signal transduction [15]. It has been shown that Gal-3 is expressed in many cell types, including endometrial cells and trophoblast cells [16-19]. Previous studies have reported that Gal-3 is specifically expressed in endometrial cells in the secretory phase [10, 20], in placental tissue during early pregnancy, and in decidua surrounding the site of implantation. Due to these findings, we speculated that the expression of Gal-3 might be aberrant in endometriosis patients with infertility.

Additionally, Gla-3 has been known as a component of a nuclear and cytoplasmic complex, shuttling between the nucleus and cytoplasm [21]. Estrogen and progesterone belong to the nuclear hormone superfamily transcription factors, which play important role in the formation of receptive endometrium. Furthermore, it has been demonstrated that endometriosis-related infertility is associated with abnormal sex hormone regulation [22]. Thus, it would be interesting to explore whether the expression of Gal-3 is regulated by sex hormones in secondary infertility of endometriosis patients.

\section{Materials and methods}

\section{Tissue collection}

We collected endometrial tissue samples from 34 women, aged 28-35 years (mean age $31.51 \pm 3.52$ ), who consulted for infertility and were found to have surgically and histologically-confirmed endometriosis. As controls, we also collected endometrial biopsy specimens from 34 healthy women aged 30-35 years (mean age $32.55 \pm 3.90$ ). Women in the control group underwent tubal sterilization and were laparoscopically confirmed to be free of endometriosis. These women displayed normal menstrual cycles and had not received anti-inflammatory or hormonal therapy within 3 months prior to surgery. The phase of the subjects' menstrual cycle was determined according to the criteria of Noyes et al. [23].
The endometrial tissue samples from each group were digested for cell culture, snap frozen and kept under $-80{ }^{\circ} \mathrm{C}$ for subsequent real-time PCR and western blot analysis, or fixed in $4 \%$ paraformaldehyde for paraffin blocks. Informed consent was obtained in writing from all subjects before surgery.

\section{Cell culture}

Primary proliferative phase endometrial stromal and glandular epithelial cells were obtained from six subjects in the endometriosis group and six subjects in the control group. For each subject, secretory phase endometrial stromal and glandular epithelial cells were also obtained. Endometrial tissue was transported from the site of collection to the laboratory in Hanks' balanced salt solution. The tissue was then minced and digested in Hanks' balanced salt solution containing $1 \%$ penicillin, $1 \%$ streptomycin, $5 \%$ collagenase, and $0.5 \%$ deoxyribonuclease at $37{ }^{\circ} \mathrm{C}$ for $30 \mathrm{~min}$ with agitation. The dispersed endometrial cells were separated by filtration through a wire sieve $(73-\mathrm{mm}$ diameter pore, Sigma). The endometrial glands (largely undispersed) were retained by the sieve, whereas the dispersed stromal cells passed through the sieve into the filtrate. Endometrial glandular epithelial cells (EECs) were plated on Madrigal coated 12 well-plates, while endometrial stromal cells (ESCs) were plated on plastic 12-well plates. Both types of cells were plated in DMEM/F12 phenol red medium (Gibco Invitrogen,Carlsbad, CA, USA) containing $10 \%$ fetal bovine serum (FBS) (Gibco). Cell cultures were maintained at $37{ }^{\circ} \mathrm{C}$ in a humidified atmosphere $\left(5 \% \mathrm{CO}_{2}\right)$ and were allowed to replicate to confluence. Thereafter, cells were passaged by standard methods of trypsinization and allowed to replicate to confluence, which required approximately $24-48 \mathrm{~h}$. Cells after first passage were characterized as described previously [24].

\section{Real-time PCR analysis}

Total RNA was extracted from 34 samples from the endometriosis group and 34 samples from the control group using Trizol Reagent (Invitrogen). Total RNA ( $1 \mu \mathrm{g})$ was reverse transcribed using a PrimeScript RT reagent kit (Takara, Dalian, China). Reverse-transcription PCR was performed prior to quantitative real-time PCR. The mRNA levels were determined by real-time PCR using SYBR Premix Ex Taq (TaKaRa) with the Applied Biosystems 7000 system SDS software as previous described [25]. Glyceraldehyde 3-phosphate dehydrogenase (GAPDH) was used as an endogenous control to normalize for differences in the amount of total RNA in each sample. The primer sequences and the sizes of the amplified fragments were as follows: Gal-3 (93 bp) 5'-CTT CCA CTT TAA CCC ACG CTT 
CAA-3' (sense), 5'-TGT CTT TCT TCC CTT CCC CAG TTA TT-3' (anti-sense); GAPDH (131 bp) 5'-ATG ACC CCT TCA TTG ACC-3' (sense), 5'-GAA GAT GGT GAT GGG ATT TC-3' (anti-sense).

\section{Immunohistochemistry}

The paraffin blocks were cut into sections of $4 \mu \mathrm{m}$, mounted on polylysine-coated microslides, dewaxed, and rehydrated. Then, tissue sections were incubated in $3 \%$ hydrogen peroxide at room temperature. For antigen retrieval, tissue slides were immersed in citric acid and boiled in a microwave oven. The sections were washed in distilled water and phosphate buffered saline (PBS, Gibco), and then subjected to bovine serum to block unspecific binding agents. This step was followed by overnight exposure $\left(4{ }^{\circ} \mathrm{C}\right)$ to the mouse anti-human Gal-3 antibody (mab1154, R\&D Systems Inc., Minneapolis, MN, USA) in a humidified chamber. After being rinsed in PBS, the sections were incubated with the bridging rabbit anti-mouse immunoglobulins conjugated with horseradish peroxidase (HRP)-labelled dextran polymer for $1 \mathrm{~h}$. After washing in PBS, diaminobenizidine tetrahydrochloride (DAB) solution was applied, followed by running tap water as well as nuclear counterstaining with haematoxylin (Sigma, USA). The stained slides were viewed under microscopy (OLYMPUS OPTICAL CO., Ltd.) under $400 \times$ magnification. Positive cells were characterized by the brown staining of Gal-3 antibody. The intensity and distribution of the staining reaction were evaluated by two blinded, independent observers.

\section{Western blot}

Protein samples were extracted from tissues or cells, and the protein concentration was measured by bicinchoninic acid assay (BCA). Samples were run in SDS-PAGE gel, transferred to nitrocellulose membranes, and immunoblotted overnight with gentle shaking at $4{ }^{\circ} \mathrm{C}$ with primary antibodies. The primary antibodies included monoclonal mouse anti-human Gal-3 antibody (1:2500) and monoclonal mouse anti-human GAPDH (Kangchen, Shanghai, China) antibody (1:5000). This procedure was followed by incubation with horseradish peroxidase-conjugated secondary antibodies. The reactions were detected by enhanced chemiluminescence assay. GAPDH was used as an endogenous control for normalization.

\section{Hormonal stimulation protocol}

For the hormone stimulation test, $5 \times 10^{5}$ cells in $1 \mathrm{ml}$ of media were plated on a 6-well plate and grown for $24 \mathrm{~h}$ in normal medium containing $10 \%$ FBS without antibiotics.
Then, the media were replaced by serum-free and phenol red-free culture media for $24 \mathrm{~h}$ to prepare for the stimulation experiment. Stimulation was performed under various concentrations of $17 \beta$-estradiol $\left(\mathrm{E} 2,0,10^{-10}-10^{-6} \mathrm{M}\right.$, Sigma) alone and under various concentrations of progesterone ( $\mathrm{P} 4,0,10^{-9}-10^{-5} \mathrm{M}$, Sigma) alone. The same experiment was performed with E2, P4, or E2 combined with P4 (E2P4, $10^{-8}$ M E2, $10^{-7}$ M P4, Sigma) for 24, 48, and $72 \mathrm{~h}$. The control was plated in phenol red-free culture media containing charcoal-stripped $10 \%$ FBS (Bioind, Shanghai, China).

\section{Statistical analysis}

Data were expressed in terms of mean \pm SEM. One-way ANOVA analyses were performed and least significant difference (LSD) tests were applied for post hoc testing using SPSS software version 15.0 with $p<0.05$ considered statistically significant.

\section{Results}

\section{Gal-3 expression in endometria of endometriosis and control groups}

To verify whether expression of Gal-3 is abnormal in the endometria of the endometriosis group, we performed immunochemistry analysis to display the expression pattern of Gal-3 proteins in endometria from both the healthy and endometriosis group (Fig. 1). Our results showed that Gal-3 was presented in the endometrium from both the control group (Fig. 1b) and the endometriosis group (Fig. 1c).

Then, Gal-3 mRNA expression levels in all endometrium samples were detected by real-time PCR analysis. As shown in Fig. 2a, Gal-3 showed significantly higher levels of expression in the secretory phase than the proliferative phase, regardless of group. Moreover, during both phases, Gal-3 expression was significantly down-regulated (secretory stage $0.47 \pm 0.02$ vs $0.05 \pm 0.01, p<0.05$; proliferative phase $0.26 \pm 0.02$ vs $0.02 \pm 0.01, p<0.05$ ) in eutopic endometrium from endometriosis patients compared to the normal endometrium. This result was confirmed at the protein level by western blot analysis (Fig. 2b).

\section{Gal-3 expression in epithelial cells and stromal cells of endometria}

The endometrium becomes receptive to embryonic attachment in the secretory phase of the menstrual cycle; thus, we explored whether Gal-3 expression is different in EECs and ESCs during this phase. The results of realtime PCR showed that Gal-3 expression was significantly 

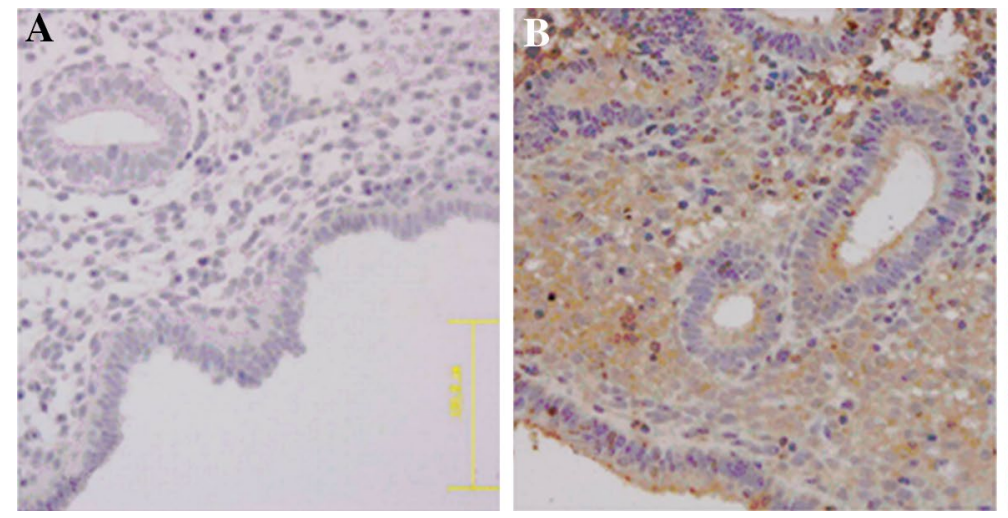

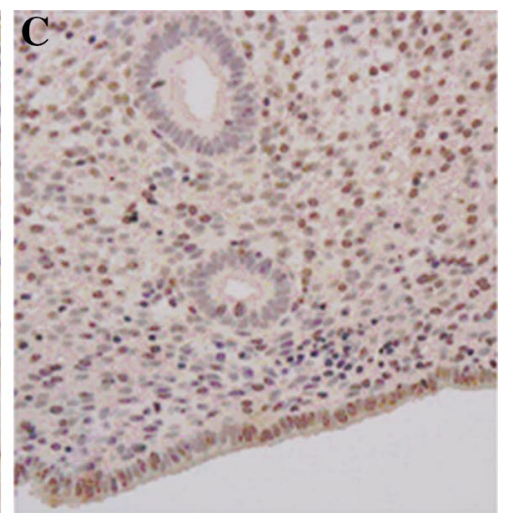

Fig. 1 Immunohistochemistry analysis of Gal-3 expression. a Negative control. b Secretory phase endometrium from healthy controls. c Secretory phase eutopic endometrium from endometriosis patients
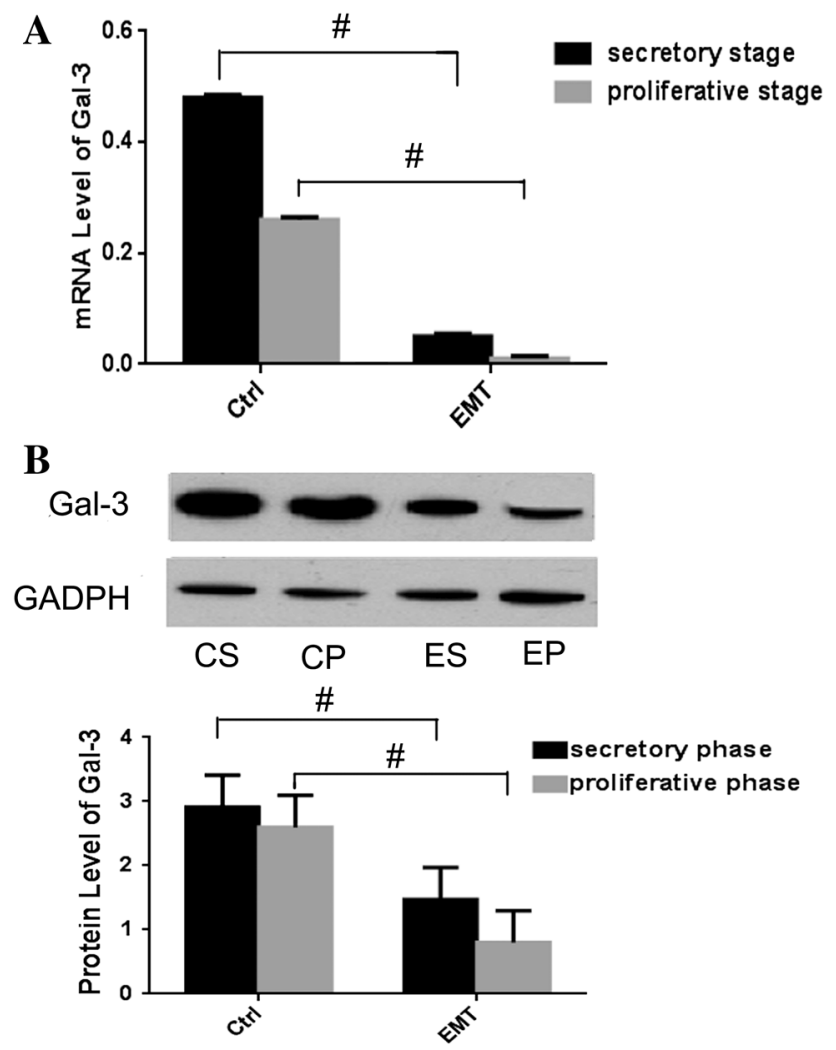

Fig. 2 Gal-3 mRNA and protein expression in endometria samples from patients and healthy women. a Relative expression levels of Gal-3 mRNA in different samples measured by real-time PCR analysis. b Relative expression levels of Gal-3 protein in different samples measured by western blot analysis. Expression levels of Gal-3 were normalized against those of GAPDH in matched samples. $E S$ endometria of patient with endometriosis in secretory phase, EP endometria of patient with endometriosis in proliferative phase, $C S$ endometria of controls in secretory phase, $C P$ endometria of controls in proliferative phase, Control control group, EMT endometriosis group; ${ }^{\#} p<0.05$ with infertility. Mouse IgG was used in the negative control (a), while Gal-3 antibody was used other analyses $(\mathbf{b}, \mathbf{c})$

down-regulated in EECs from the endometriosis group compared to the healthy group $(0.14 \pm 0.06$ vs $0.59 \pm 0.07$, $p<0.05$ ) (Fig. 3a). In contrast, there was no significant change in Gal-3 expression in ESCs from the control group compared to the endometriosis group; however, there was a trend of down-regulation of Gal-3 in the endometriosis group. We further confirmed these results by western blot analysis. As shown in Fig. 3b, a significant down-regulation of Gal-3 protein was observed in EECs but not ESCs of eutopic endometrial samples from the endometriosis group compared to the control group $(0.22 \pm 0.05$ vs $0.58 \pm 0.07$, $p<0.05)$.

\section{Hormone-stimulated Gal-3 expression in endometrial epithelial cells}

To further investigate the hormone regulation of Gal-3 expression in EECs, various concentrations of E2 and P4 were used to co-culture EECs from the control group for $24 \mathrm{~h}$ before mRNA levels of Gal-3 were detected. Our results showed that Gal-3 was up-regulated by E2 and $\mathrm{P} 4$ at all concentrations, reaching peak level at $10^{-8}$ and $10^{-7} \mathrm{M}$, respectively (Fig. 4a, b) Thus, concentrations of $10^{-8} \mathrm{M}$ of E2 and $10^{-7} \mathrm{M}$ of $\mathrm{P} 4$ were used in the subsequent experiments.

Then, the expression of Gal-3 was detected in EECs pretreated with $10^{-8} \mathrm{M} \mathrm{E} 2,10^{-7} \mathrm{M} \mathrm{P} 4$, or $10^{-8} \mathrm{M}$ E2 combined with $10^{-7} \mathrm{M}$ P4 for 24,48 , and $72 \mathrm{~h}$, respectively. In the control group, Gal-3 expression was induced by E2 or P4 treatment in a time dependent manner (Fig. 5a). Expression of Gal-3 in the E2P4 group was lower than that in the P4 treatment group and higher than that in the E2 treatment group. These results suggest Gal-3 induction is more sensitive to P4 than E2 treatment; however, in EECs from the eutopic endometria with endometriosis (Fig. 5b), Gal-3 expression increased slightly after hormone pretreatment 


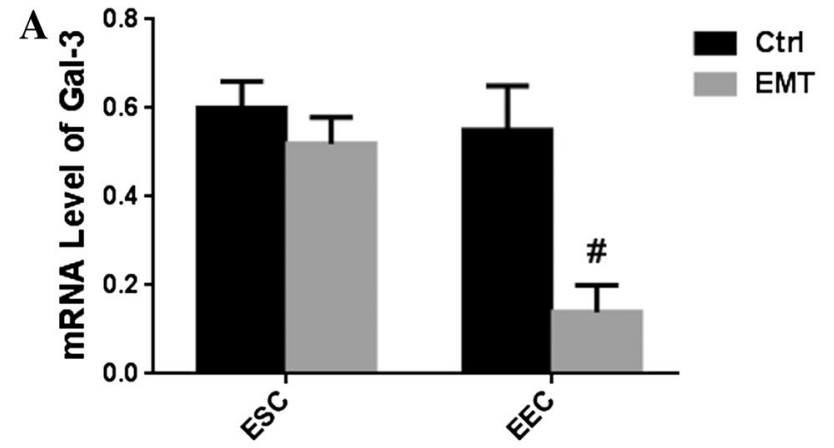

B
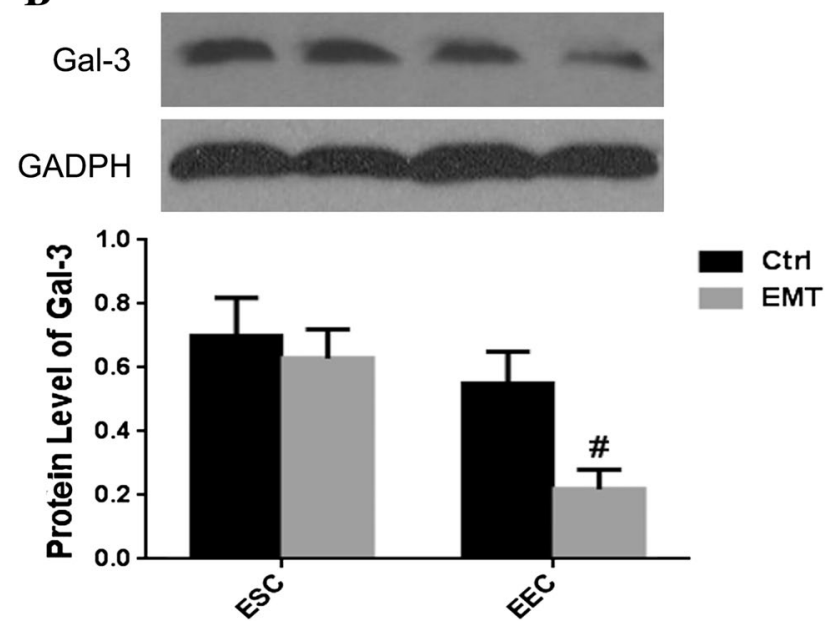

Fig. 3 Expression of Gal-3 mRNA and protein in epithelial cells and stromal cells from secretory phase endometria. a Relative expression levels of Gal-3 mRNA in EECs and ESCs. b Relative expression levels of Gal-3 protein in EECs and ESCs; $C t r l$ control group, EMT endometriosis group, EECs endometrial glandular epithelial cells, ESCs endometrial stromal cells; ${ }^{*} p<0.05$

for $24 \mathrm{~h}$, and then decreased time-dependently. Furthermore, compared with the control group, the expression of Gal-3 was lower in the endometriosis groups at all time points.

\section{Discussion}

Endometriosis shows a serious impact on female fertility, but the etiology and pathogenesis of endometriosis-related infertility are unknown. Thus, it is imperative to identify the molecular mechanism of endometriosis to develop an effective therapy for endometriosis patients with infertility. Several studies have reported that endometriosis is a major cause of infertility due to its adverse effect on endometrial receptivity to embryonic implantation [26].

Our previous study verified that Gal-3 plays an important role in the process of embryonic implantation [25]. Intracellular Gal-3 promoted proliferation and adhesion in endometrial cells. Decreased expression of Gal-3
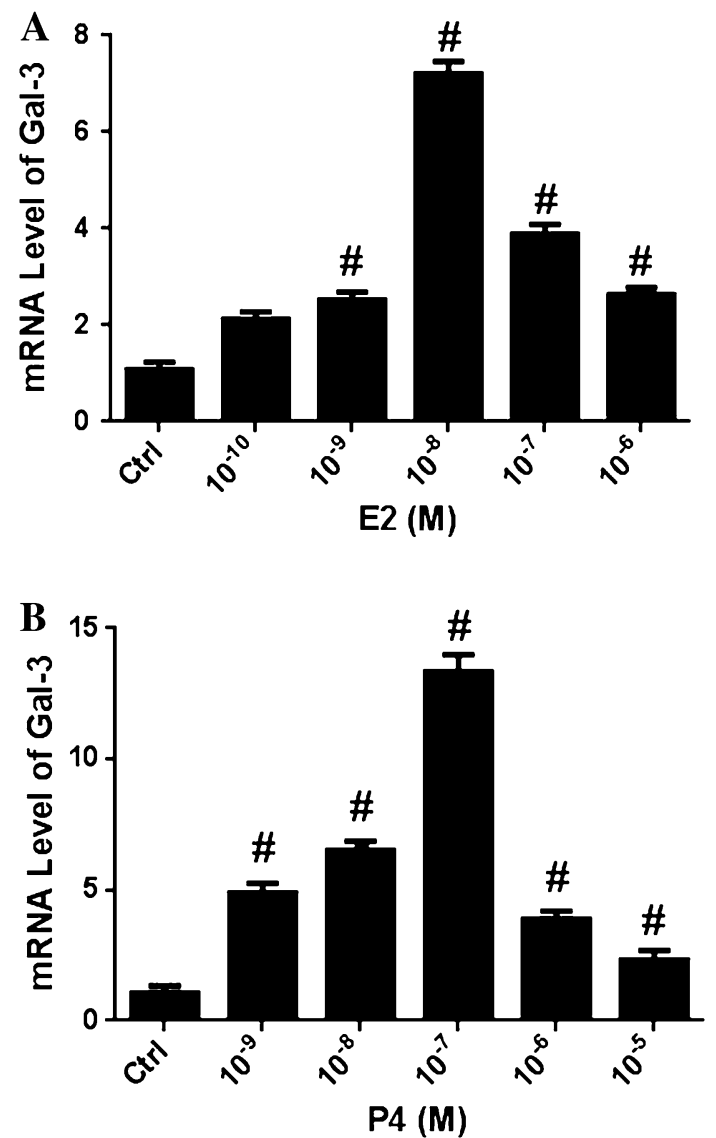

Fig. 4 Hormonal regulation of Gal-3 expression by E2 and P4 in Endometrial epithelial cells. EECs were treated with E2 (a) (Ctrl, $\left.10^{-10}-10^{-6} \mathrm{M}\right)$ and P4 (b) (Ctrl, $\left.10^{-9}-10^{-5} \mathrm{M}\right)$ for $24 \mathrm{~h}$, mRNA level of Gal-3 was determined by real-time PCR. Gal-3 expression was analyzed by real-time PCR and GAPDH was used as an endogenous control to normalize for differences. EECs endometrial glandular epithelial cells; ${ }^{\#} p<0.05$

hindered embryonic adhesion to endometrial epithelial cells and delayed proliferation of endometrial stromal cells in achieving optimal status to accommodate the invading embryo, resulting in failed embryonic implantation. Secreted Gal-3 inhibited cell proliferation and induced apoptosis of endometrial cells [27]. This study shows that Gal-3 is expressed in the endometrium of both endometriosis and healthy women, but is reduced significantly in the former. This suggests a defect in Gal-3 expression occurs in eutopic endometrium from endometriosis patients with infertility. Decreased Gal-3 expression in eutopic endometrium from patients with endometriosis may contribute to the defective formation of receptive endometrium, thus leading to infertility.

Hormonal regulation of cellular function impacts many dynamic biological changes occurring during the periimplantation stage of the menstrual cycle. Estrogen and progesterone act coherently at certain time intervals to stimulate 

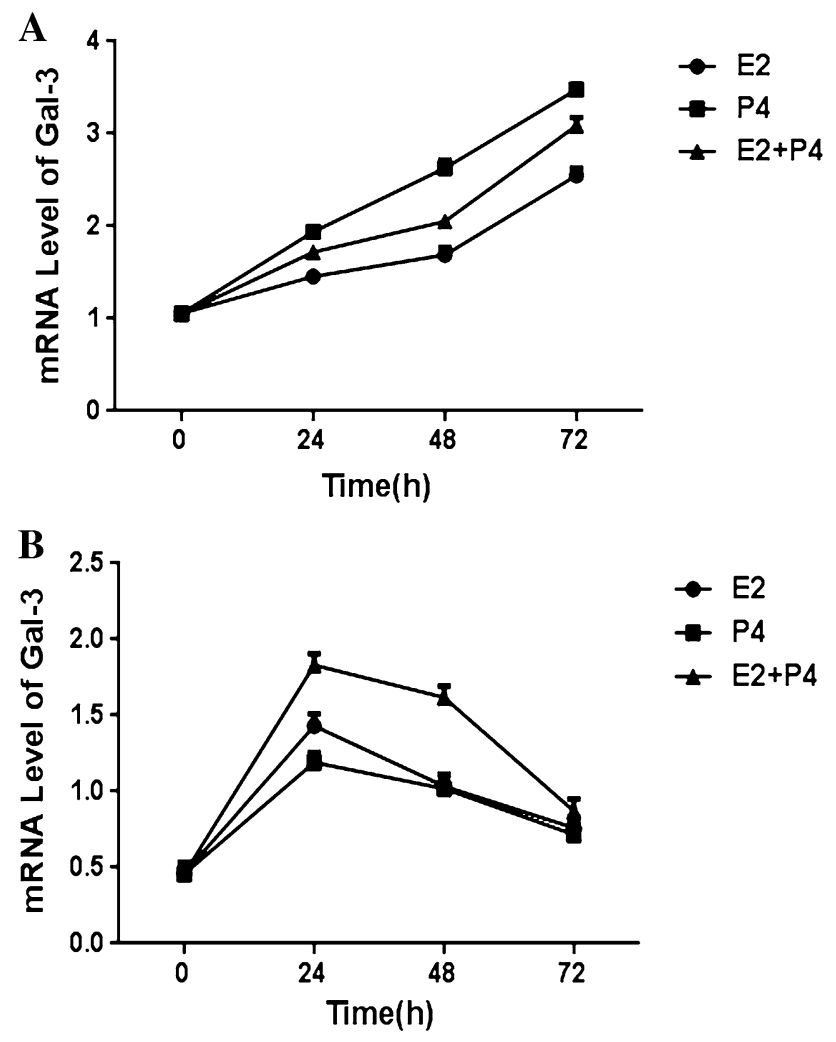

Fig. 5 Defective progesterone regulation in endometrial epithelial cells from endometriosis women with infertility. EECs from control group (a) and endometriosis group (b) were treated with $10^{-8} \mathrm{M} \mathrm{E} 2$ with or without $10^{-7} \mathrm{M} \mathrm{P} 4$ and Gal-3 expression was analyzed at 0 , $24,48,72 \mathrm{~h}$. Gal-3 expression was analyzed by real-time PCR and GAPDH was used as an endogenous control to normalize for differences. EECs endometrial glandular epithelial cells; ${ }^{*} p<0.05$

the expression of key molecules that regulate endometrial receptivity. Our results showed that Gal-3 expression specifically increased during the secretory phase of the menstrual cycle in both groups, indicating that Gal-3 may be regulated by sex hormones. To confirm this relationship, we investigated the effect of hormones on Gal-3 expression in both EECs and ESCs. We found that regulation of Gal-3 expression by $\mathrm{E} 2$ and $\mathrm{P} 4$ could be detected in EECs but not ESCs. This result indicates that Gal-3 may primarily contribute to the dynamic change of EECs during embryonic implantation. Then, we explored the physiological dose of E2 $\left(10^{-8} \mathrm{M}\right)$ and P4 $\left(10^{-7} \mathrm{M}\right)$ that maximized Gal-3 expression in EECs. The results suggested that Gal-3 is regulated by sex hormones, which is in agreement with our previous study [27]. In the control group, expression of Gal-3 was significantly increased when induced by $\mathrm{P} 4$ alone, compared to E2 alone or E2P4. From these results, we concluded that
E2, to some degree, antagonized the increased expression of Gal-3 by $\mathrm{P} 4$ in normal endometrium. In the endometriosis group, E2 alone, $\mathrm{P} 4$ alone, nor E2P4 could increase Gal-3 expression to the level of the control group. This indicates that there is no induction of Gal-3 expression in response to E2 or P4 treatment in the endometriosis group. Previous reports have shown that progesterone resistance is one important factor for endometriosis. Moreover, Gal-3 can be detected in the peritoneal fluid of endometriosis patients [28-30]; thus, defective progesterone regulation in endometriosis women with infertility might account for decreased Gal-3 expression in eutopic endometrium.

In summary, we found decreased expression of Gal-3 in eutopic endometrium from endometriosis, which may account for the defective formation of receptive endometrium. We further showed that Gal-3 was regulated mainly by hormones in EECs. We also suggested that the failure of Gal-3 elevation by hormones in EECs from endometriosis patients may contribute to progesterone resistance in endometriosis-related infertility. Although our study preliminarily indicates that the defective expression of Gal-3 may contribute to infertility in patients with endometriosis, further research is needed to detail the pathways of Gal-3 in eutopic endometrium from endometriosis.

Acknowledgments This study was supported by the National Natural Science Foundation of China (Grant No. 81300467), the Program of Shanghai Subject Chief Scientist (Grant No. 12XD1401200), and the Programs Foundation of Ministry of Education of China (Grant No. 20120071110074).

\section{Compliance with ethical standards}

Conflict of interest The authors declare no conflict of interest.

Ethical approval Consent forms and protocols were approved by the Ethical Committee of Obstetrics and Gynecology Hospital at Fudan University. All procedures performed in studies involving human participants were in accordance with the ethical standards of the institutional and/or national research committee and with the 1964 Helsinki declaration and its later amendments or comparable ethical standards. This article does not contain any studies with animals performed by any of the authors.

Informed consent Informed consent was obtained from all individual participants included in the study.

Open Access This article is distributed under the terms of the Creative Commons Attribution 4.0 International License (http://creativecommons.org/licenses/by/4.0/), which permits unrestricted use, distribution, and reproduction in any medium, provided you give appropriate credit to the original author(s) and the source, provide a link to the Creative Commons license, and indicate if changes were made. 


\section{References}

1. Giudice LC, Kao LC (2004) Endometriosis. Lancet 364:1789-1799

2. Gao X, Outley J, Botteman M et al (2006) Economic burden of endometriosis. Fertil Steril 86:1561-1572

3. Cramer DW, Missmer SA (2002) The epidemiology of endometriosis. Ann N Y Acad Sci 955:11-22 (discussion 34-16, 396-406)

4. Meuleman C, Vandenabeele B, Fieuws S et al (2009) High prevalence of endometriosis in infertile women with normal ovulation and normospermic partners. Fertil Steril 92:68-74

5. Browne H, Taylor H (2006) HOXA10 expression in ectopic endometrial tissue. Fertil Steril 85:1386-1390

6. Fazleabas AT, Brudney A, Chai D et al (2003) Steroid receptor and aromatase expression in baboon endometriotic lesions. Fertil Steril 80(Suppl 2):820-827

7. Zanatta A, Pereira RM, Rocha AM et al (2015) The relationship among HOXA10, estrogen receptor $\alpha$, progesterone receptor, and progesterone receptor $\mathrm{B}$ proteins in rectosigmoid endometriosis: a tissue microarray study. Reprod Sci 22:31-37

8. da Silva CM, Vilaça Belo A, Passos Andrade S et al (2014) Identification of local angiogenic and inflammatory markers in the menstrual blood of women with endometriosis. Biomed Pharmacother 68:899-904

9. Carmona F, Chapron C, Martínez-Zamora MÁ et al (2012) Ovarian endometrioma but not deep infiltrating endometriosis is associated with increased serum levels of interleukin- 8 and interleukin-6. J Reprod Immunol 95:80-86

10. von Wolff M, Wang X, Gabius HJ et al (2005) Galectin fingerprinting in human endometrium and decidua during the menstrual cycle and in early gestation. Mol Hum Reprod 11:189-194

11. Du GP, Zhang W, Wang L et al (2007) Identification of differentially expressed genes in endometrium during the window of implantation using suppression substractive hybridization. Zhonghua Fu Chan Ke Za Zhi 42:187-191

12. Barondes SH, Cooper DN, Gitt MA et al (1994) Galectins. Structure and function of a large family of animal lectins. J Biol Chem 269:20807-20810

13. Panjwani N (2014) Role of galectins in re-epithelialization of wounds. Ann Transl Med 2:89. doi:10.3978/j. issn.2305-5839.2014.09.09

14. Rabinovich GA, Liu FT, Hirashima M (2007) An emerging role for galectins in tuning the immune response: lessons from experimental models of inflammatory disease, autoimmunity and cancer. Scand J Immunol 66:143-158

15. Merlin J, Stechly L, de Beaucé S et al (2011) Galectin-3 regulates MUC1 and EGFR cellular distribution and EGFR downstream pathways in pancreatic cancer cells. Oncogene 30:25142525. doi:10.1038/onc.2010.631
16. Brustmann H, Riss D, Naude S (2003) Galectin-3 expression in normal, hyperplastic, and neoplastic endometrial tissues. Pathol Res Pract 199:151-158

17. Stewart CJ, Crook ML (2010) Galectin-3 expression in uterine endometrioid adenocarcinoma: comparison of staining in conventional tumor glands and in areas of MELF pattern myometrial invasion. Int J Gynecol Pathol 29:555-561

18. Dange MC, Srinivasan N, More SK et al (2014) Galectin-3 expressed on different lung compartments promotes organ specific metastasis by facilitating arrest, extravasation and organ colonization via high affinity ligands on melanoma cells. Clin Exp Metastasis 31:661-673

19. Hu R, Jin H, Zhou S et al (2007) Proteomic analysis of hypoxiainduced responses in the syncytialization of human placental cell line BeWo. Placenta 28:399-407

20. Du GP, Zhang W, Wang L et al (2006) Expression of galectin-3 in human endometrium. Fudan Univ J Med Sci 02:143-146

21. Davidson PJ, Davis MJ, Patterson RJ et al (2002) Shuttling of galectin-3 between the nucleus and cytoplasm. Glycobiology 12:329-337

22. Yang H, Lei C, Cheng $C$ et al (2012) The antiapoptotic effect of galectin-3 in human endometrial cells under the regulation of estrogen and progesterone. Biol Reprod 87:39. doi:10.1095/ biolreprod

23. Noyes RW, Hertig AT, Rock J et al (1975) Dating the endometrial biopsy. Am J Obstet Gynecol 122:262-263

24. Khan KN, Masuzaki H, Fujishita A et al (2005) Interleukin6-and tumour necrosis factor alpha-mediated expression of hepatocyte growth factor by stromal cells and its involvement in the growth of endometriosis. Hum Reprod 20:2715-2723

25. Lei CX, Zhang W, Zhou JP et al (2009) Interactions between galectin-3 and integrinbeta3 in regulating endometrial cell proliferation and adhesion. Hum Reprod 24:2879-2889

26. Giudice LC, Kao LC (2004) Endometriosis. Lancet 364:1789-1799

27. Yang H, Taylor HS, Lei C et al (2011) Hormonal regulation of galectin 3 in trophoblasts and its effects on endometrium. Reprod Sci 18:1118-1127

28. Fazleabas AT (2006) A baboon model for inducing endometriosis. Methods Mol Med 121:95-99

29. Lessey BA, Palomino WA, Apparao KB et al (2006) Estrogen receptor-alpha (ER-alpha) and defects in uterine receptivity in women. Reprod Biol Endocrinol 4(Suppl 1):S9

30. Caserta D, Di Benedetto L, Bordi G et al (2014) Levels of galectin-3 and stimulation expressed gene 2 in the peritoneal fluid of women with endometriosis: a pilot study. Gynecol Endocrinol 29:1-4 